\title{
Modeling of Football Match Outcomes with Expected Goals Statistic
}

\author{
Adan Partida ${ }^{1}$, Anastasia Martinez ${ }^{1}$, Cody Durrer ${ }^{1}$, Oscar Gutierrez $^{1}$ and Filippo Posta ${ }^{1}$ \\ ${ }^{1}$ Estrella Mountain Community College, Avondale, AZ, USA \\ DOI: https://doi.org/10.47611/jsr.v10i1.1116
}

\section{ABSTRACT}

\begin{abstract}
Aim. Our research examined the predictive capabilities of mathematical models that are solely based on the expected goal statistics obtained from a publicly available database.

Method. We collected match and expected goals data for 310 matches from three European Leagues (Bundesliga, La Liga, and Serie A). We created three probabilistic models based on the expected goals statistic and compared them with two well-established probabilistic models using binomial deviance, squared error, and profitability in the betting market as evaluation metrics.

Results. Our best model adjusted the expected goal statistics for homefield advantage and outperformed the two probabilistic models used for comparison. Two of our models were profitable under certain betting conditions.

Limitations. Our models explored a simplistic integration of expected goals into a Poisson based probabilistic model and did not include other contributing factors such as a team's defensive prowess. The number of games simulated was also limited due to the premature closure of the European Leagues due to the COVID-19 pandemic.

Conclusions. The use of a probabilistic model based solely on expected goals score statistic can provide some meaningful insight into forecasting the outcome of a football match and can develop useful betting strategies.
\end{abstract}

\section{Introduction}

Modern engineering and computer science allow for the accumulation of relevant measurements across many fields of interest. These measurements are combined into datasets that are mined to identify patterns used to answer field specific questions. Data analytics has become fundamental in all aspects of the sports industry (Mondello \& Kamke 2014). Sport organizations use predictive analytics to improve their odds on the court, injury prevention, ticket pricing, and payroll optimization (Link, 2018; Dobson et al., 2001). Even governing bodies, such as the Federation Internationale de Football Association (FIFA), use data analytics to determine rankings in seeding decisions that can have major financial implications (Monks \& Husch, 2009). The betting industry is another industry that has been at the forefront of the data analytics revolution. Oddsmakers use data to improve the calculations of odds but also to provide new betting opportunities for their customers such as live betting (Pearson, 2017). The sheer economical size of the sports and betting industries has created a fertile and extremely competitive ground for scientists to create new ways to measure hard to quantify features (e.g., effort, clutch) and to develop predictive models that can lead to economical and on-court advantages (Cintia et al., 2015; Link, 2018). These new data and predictive models use approaches from many fields of science, from engineering to finance and medicine, and often lead to extremely advanced and complex algorithms.

As the most global sport, football (soccer in the US) is an excellent case study for the analysis and development of sports analytics. The game has been studied in depth for many years by fans and scholars alike and there are many publicly available databases that can be used as resources for game data (Rein \& Memmert, 2016; Cintia et al., 
2015; Link, 2018). The football betting industry is also a well-established worldwide phenomenon grossing billions of dollars.

Interest in predicting football match outcomes is matching the popularity of the sport and can be summarized into two overlapping trends: creating new statistics and developing computational algorithms that take advantage of the throve of available data. One of the most promising statistics is expected goals (xG). $x G$ was first introduced for professional ice hockey (Macdonald, 2012) and has since become ubiquitous in football (Eggels et al., 2016). xG data are often used as one of the features of interest to create computational models involving advanced data science techniques such as artificial neural networks (Hucaljuk \& Rakipovic, 2011) or in combination with other factors (e.g., payroll, match attendance, etc.) to create sophisticated rating systems (www.fivethirtyeight.com).

Our study explored the possibility of using publicly available data to create straightforward mathematical models to predict the most likely outcome of a football match. Our analytical modeling approach can be applied to any football match. For practicality, we collected data from 3 European leagues (German "Bundesliga", Italian "Serie A", and "Spanish Liga"). The models are straightforward because they are based off the well-known football principle that it is harder to score than to defend. Therefore, our models focus on predicting goals by team. In our approach, goals scored by a team are predicted almost independently from a team's opposition. We only used game related data instead of trying to incorporate too many variables such as form, injuries, line-up, game importance, etc.

To assess the quality of our models, we used the probabilities used by Las Vegas oddsmakers as well as a well-established model from the 538 website (www.fivethirtyeight.com) as "gold standards" for comparison. In addition to studying our models' predictive capabilities, we also analyzed if the models could be profitable under some simple betting rules and compared this profitability with the gold standards.

The idea behind this approach to predicting football math outcomes is not new. Our first model in this study is a straightforward starting point in the implementation of any xG based predictive model. The novelty of our study is based on the investigation of simple changes to the basic model and how they compare to more advanced models, as well as the use of publicly available data that which allow for ease of replication and further exploration.

\section{Materials and Methods}

We collected publicly available data to create simple but highly interpretable statistical models and we also used publicly available data to compare the predictive abilities of our models. The workflow of our study is summarized in Figure 1 and describe in more details below. 


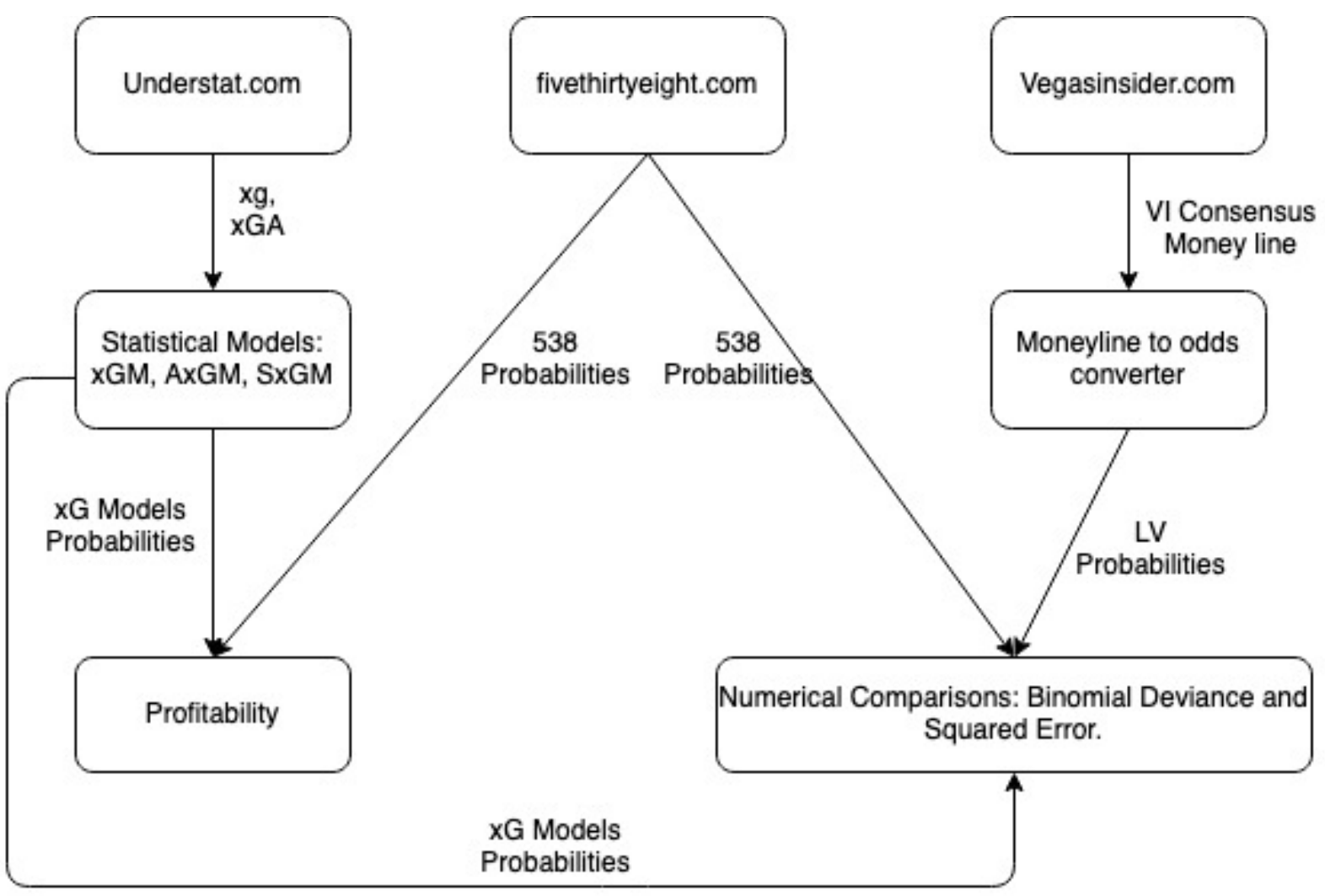

Figure 1 - Workflow of the study from data collection to qualitative comparison and profitability.

\section{Dataset}

We built a Microsoft Excel spreadsheet of football data during the 2019/20 football seasons for three European leagues: Bundesliga (Germany), La Liga (Spain), and Serie A (Italy). We wanted to use publicly available data including the $\mathrm{xG}$ statistic derived solely from physical football events. We selected the website www.understat.com (Understat) for their "detailed XG statistics trained using neural networks on more than 100,000 shots". Understat uses on-field statistics of players such as shots, passes, goals, appearances and minutes played to build the xG statistic. From Understat, we collected weekly team fixtures including team names, $\mathrm{xG}$ scored $(\mathrm{xG})$ and allowed (xGA) for each team in the league. We also collected the number of matches (M) played by each team at the time of collection. We collected this data on a weekly basis beginning on Week 7 of each league and continuing until the league suspension due to the COVID-19 pandemic. For comparison purposes, we collected the predictive match probabilities created by 538 for the same fixtures in our dataset. Finally, we collected match odds from the Vegas Insider website (www.vegasinsider.com). Within the website, we picked the odds in the "VI Consensus" column. These odds consist of the betting line that occurs most frequently among Las Vegas and Global bookmakers followed by Vegas Insider. We used Understat's data to build our predictive models and the data from 538 and LV to compare the predictive capabilities of our models. The final spreadsheet contained 310 matches from the 3 European Leagues.

\section{Predictive Models}

$x G$ Model $(x G M)$ - Our starting model used two assumptions: that the best predictor of the numbers of goals is the $\mathrm{xG}$ statistic, and that the number of goals scored by a team follows a Poisson distribution. xG represents the number of goals a football team is expected to score in a match against an opponent based on the quantity and quality of chances created (Eggels et al., 2016). 
We used the $\mathrm{xG}$ statistic as input for the Poisson distribution to generate goal scoring probabilities that were combined into probabilities of a home, away, or draw outcome. The Poisson distribution provides a valid approximation capable of representing the number of goals scored in a football match (Karlis \& Ntzoufras, 2003).

The xGM model calculates the probability of a team scoring a certain number of goals by using the following Poisson formula:

$$
P(k)=e^{-x G} \frac{x G^{k}}{k !}
$$

where $\mathrm{P}(k)$ is the Poisson probability that exactly k goals are scored in a match.

The process of building the probability of having a home win, away win or draw from this model is depicted in Figure 1. The figure uses as example a match in the Bundesliga between Eintracht Frankfurt (home team) and Bayern Munich (away team). For each team, we calculated $\mathrm{P}(\mathrm{k})$ with $\mathrm{k}$ going from 0 to $5+$ (the 5 goals or more case being 1 minus the sum of all of the previous probabilities) with the above formula. The home team probabilities are aligned horizontally, and the away team probabilities are aligned vertically. Each cell on the table below and to the right of the goal probabilities shows the probability of an exact score obtained by multiplying the appropriate probabilities. For example, the probability of a 1-1 draw is obtained by multiplying the probability of the home team scoring 1 goal $(\mathrm{P}(1)=0.02)$ and the probability of the away team scoring also 1 goal $(\mathrm{P}(1)=0.03)$. Therefore, the probability of Eintracht and Bayern tying 1-1 is 0.06. We calculated the probability of a draw by summing all of the probabilities where the home team and away team score the same number of goals (shaded diagonal in Figure 2). For the home and away team winning probabilities, we sum all of the probabilities for the respective outcome. We calculated the draw probability as the difference between 1 and the sum of the home and away win probabilities to account for rounding errors and to verify that the sum of the probabilities of the three outcomes is 1 .

\begin{tabular}{|c|c|c|c|c|c|c|c|c|c|c|c|}
\hline & & & & $x G$ & XAH & 1.731111111 & & & & & \\
\hline & & & & 1.731111111 & 1.47 & & & & & & \\
\hline & & & 0 goals & 1 goal & 2 goals & 3 goals & 4 goals & 5 goals & Home Team & & \\
\hline & & Probability & 0.1770875367 & 0.3065582024 & 0.2653431552 & 0.1531128281 & 0.06626382948 & 0.03163444818 & Eintracht & & \\
\hline$x G$ & 0 goals & 0.07585824415 & 0.01 & 0.02 & 0.02 & 0.01 & 0.01 & 0.00 & & & \\
\hline 2.578888889 & 1 goal & 0.195629983 & 0.03 & 0.06 & 0.05 & 0.03 & 0.01 & 0.01 & & Home Win & 0.25 \\
\hline XAA & 2 goals & 0.2522539947 & 0.04 & 0.08 & 0.07 & 0.04 & 0.02 & 0.01 & & Draw & 0.19 \\
\hline \multirow[t]{5}{*}{1.172222222} & 3 goals & 0.216845008 & 0.04 & 0.07 & 0.06 & 0.03 & 0.01 & 0.01 & & Away Win & 0.56 \\
\hline & 4 goals & 0.1398047955 & 0.02 & 0.04 & 0.04 & 0.02 & 0.01 & 0.00 & & & 1.00 \\
\hline & 5 goals & 0.1196079746 & 0.02 & 0.04 & 0.03 & 0.02 & 0.01 & 0.00 & & & \\
\hline & Away Team & & & & & & & & 1.00000 & & \\
\hline & Bayern Munich & & & & & & & & & Draw & \\
\hline
\end{tabular}

Figure 2- Sample probability matrix for the xGM. The shaded cells show the probabilities of 0-0, 1-1, 2-2, 3-3, 4-4, or $5+-5+$ draws, respectively. Their sum represents the probability that the match ends in a draw according to the xGM.

Scaled $x G$ model (SxGM) - The $x G$ model does not take into consideration the opposition. We hypothesized that a better model would include the effect of the opposing team defense. We created a scaled version of the $\mathrm{xG}$ model (SxGM). In this model, the input parameter for the Poisson probability distribution consists of a scaled version of xG. In particular, $x G$ statistics are scaled by the expected goals allowed (xGA) by the opposing team. For the SxGM the probability that a team scores $\mathrm{k}$ goals is given by:

$$
P(k)=e^{-x G * x G A_{\text {opp }}} \frac{\left(x G * x G A_{\text {opp }}\right)^{k}}{k !}
$$


Where $x G A_{\text {opp }}$ is the expected goals per game allowed by the opposing team. The match outcome probabilities are calculated analogously to the $\mathrm{xGM}$

Adjusted $x G$ model (AxGM) - In this model, the input parameter for the Poisson probability distribution consists of an adjusted version of $x G$. Expected goals statistics are adjusted by adding a coefficient $\left(k_{H F}\right)$ that represents leaguebased home-field advantage. $k_{H F}$ is calculated by taking the mean difference of $\mathrm{xG}$ overall versus $\mathrm{xG}$ at-home over the past 5 years. For this adjusted model the probability that a team scores $\mathrm{k}$ goals is given by:

$$
P(k)=e^{-\left(x G+k_{H F}\right)} \frac{\left(x G+k_{H F}\right)^{k}}{k !}
$$

To validate our choice of $k_{H F}$ we ran a mean comparison analysis of the goal distributions, we calculated $95 \%$ confidence intervals of the difference of the means, and we computed the effect sizes using Cohen's D (Cumming, 2014). All of this was done separately for each league.

\section{Gold Standards}

To test the performance of our three models we compared them to the predictions of popular statistics website 538 and the Las Vegas spreads published by Vegas Insider. 538 is an established statistical analysis website with data on over 550,000 football matches going as far back as 1888 derived from ESPN's database and the Engofootballdata GitHub repository (Jayboice, 2018). This large sample size of football match data allows 538's predictive analytics method to benefit from multiple adjustments to corresponding probabilities in their data matrix as new games are played. 538's model accounts for off-field parameters such as injuries, salaries, and match importance. Vegas Insider is a collection of spreads generated from multiple sportsbooks. We chose the VI Consensus sportsbook as the gold standard for betting as it is an aggregate of the most common betting line that appears among the Las Vegas and Global sportsbooks and therefore represents the average betting odds. Sports books such as the ones used in Las Vegas create power ratings to compute and order teams against each other using a variety of variables that go beyond onfield statistics (Kramer, 2017). Power ratings is the industry term for analytics based on game factors and are no different than the metrics derived from 538. Of course, no two statistical methods are exactly alike but keeping as many computational steps as similar as possible allows for a fairer comparison and is why our group considers 538 and Vegas Insider to be gold standards for comparison.

Note that the Vegas odds are not probabilities, but money-lines. To transform them into probabilities we used the following formula:

$$
P(\text { moneyline })= \begin{cases}\frac{100}{(100+\text { moneyline }) * \text { Vig }} & \text { if moneyline }>0 \\ \frac{- \text { moneyline }}{(100-\text { moneyline }) * \text { Vig }} & \text { if moneyline }<0\end{cases}
$$

Where money line represents the odds for either a home win, draw, or away win, and the Vig is the amount that the bookmakers charge each bettor for the "right" to place the bet. For our dataset it ranges between 1.03 and 1.07. Through the above formula we were able to obtain a decimal value matching the format of our models and 538's.

\section{Model performance metrics}

We wanted to test the predictive ability of our models as well as their ability to be profitable in the betting market. Therefore, we conducted three separate accuracy studies to compare the performance of our models among themselves and with the gold standards. One of the measures we used is the binomial deviance error across all games in each league. Binomial deviance error is given by 


$$
-\left(s_{i} \log _{10} p_{i}+\left(1-s_{i}\right) \log _{10}\left(1-p_{i}\right)\right)
$$

where $s_{i}$ is either 0 (home win), 0.5 (draw), or 1 (away win) and p represents the probability of the outcome (Lasek et al., 2013). For the example in Figure 2, the binomial deviance error if the game ended in a draw would have been $-\left(0.5 \log _{10}(0.19)+(1-0.5) \log _{10}(1-0.19)\right) \approx 0.406$.

We also calculated the squared error of the prediction by using the formula:

$$
\left(s_{i}-p_{i}\right)^{2}
$$

where the variables have the same meaning as the binomial deviance formula (Lasek et al., 2013).

Finally, we explored the profitability of our three models and 538 by incorporating three betting strategies and calculating their financial outcomes. The three strategies consisted of betting on all games by choosing the match outcome with the highest probability of success (we labeled this rule "Best" in the results section), only betting on games with a match outcome that had a probability greater than $50 \%$ (i.e., " $>50$ ") and only betting on games with an outcome that has a probability greater than $70 \%$ (i.e., " $>70 ”)$.

\section{Results \& Discussion}

We investigated the predictive abilities of our models versus the gold standards as well as their profitability. Goldstandard comparisons using binomial deviance error and the squared error of prediction from the 310 matches in the dataset are shown in Figures (3) and (4).

The AxGM model is the model with the best accuracy with respect to binomial deviance error among our models. Its mean error is $0.298 \pm 0.118$ versus the $\mathrm{xGM}$ mean binomial deviance error of $0.356 \pm 0.132$ and the SxGM mean binomial deviance error of $0.370 \pm 0.204$. These results suggest that adjusting expected goals statistics for home/away splits improves model predictive capabilities more than adjusting for the opposition's defensive capabilities. The error reduction due to adjusting for home field is about $16 \%$. In addition, taking into account the opposition's defensive abilities does not reduce the deviance error (there is actually a $1.5 \%$ increase on average). AxGM is also the best performing model when compared to the gold standards and also the one with the least variation. The 538 mean binomial deviance error of $0.302 \pm 0.145$ is about $1.2 \%$ worse on average than our best model. The Las Vegas mean binomial deviance error of $0.328 \pm 0.189$ is $9.1 \%$ worse than AxGM with a significantly higher standard deviation. The other two of the $\mathrm{xG}$ models perform worse than the gold standards.

We also looked at the accuracy in predicting individual leagues. The AxGM model performed the best for Bundesliga and Serie A, while the 538 predictions are the most accurate for the Spanish Liga. These results are shown in Table 1 and confirm the aggregate results with the interesting exception of the Italian Serie A where SxGM performs better than $\mathrm{xGM}$. This result is interesting because Italian football has always been known for its defensive prowess and SxGM tries to account for the opposition's defensive abilities. 


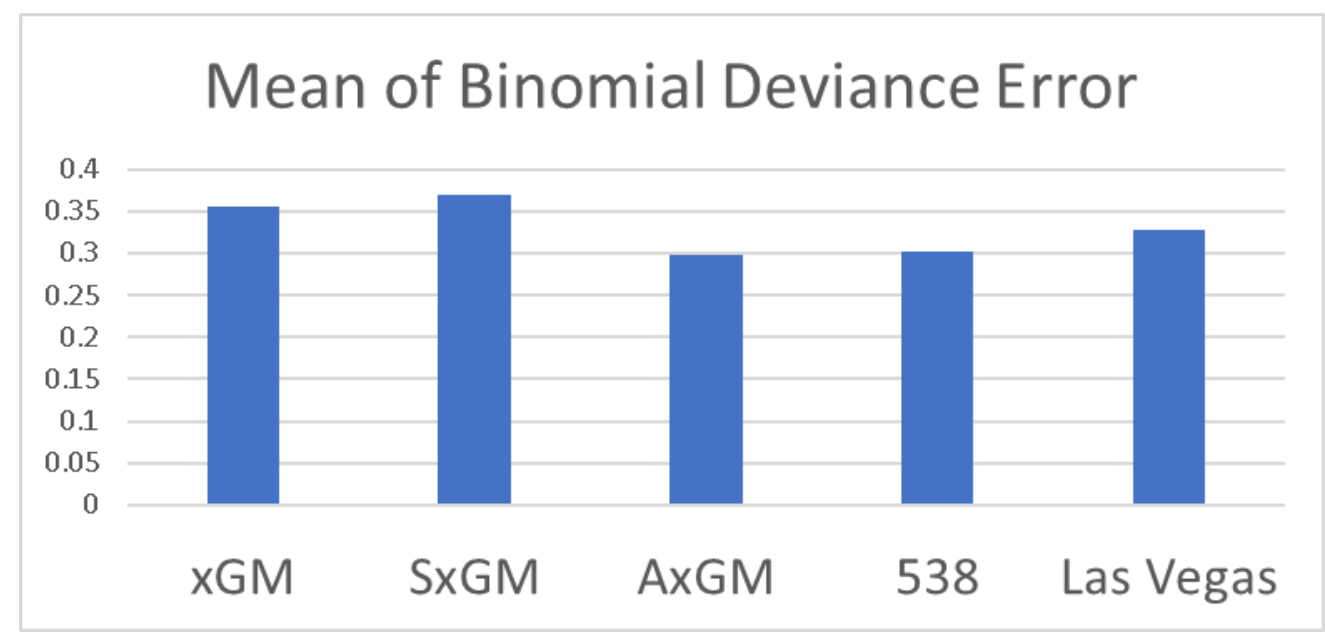

Figure 3 - Mean binomial deviance error results for our three models and the two gold standards.

We found similar error patterns for the squared error analysis. Figure 4 shows the mean squared error across the 310 games and its only difference from Figure 3 consists of the squared error for the SxGM model being lower than xGM. We also analyzed the squared error across the leagues and the results were qualitatively the same as what we found in Table 1 for the binomial deviance (data not shown).

Table 1 - Mean and standard deviations of the binomial deviance error for statistical models organized by League. The bolded values represent the least error for each league.

\begin{tabular}{|c|c|c|c|c|c|c|}
\hline \multirow[b]{2}{*}{$\mathrm{xGM}$} & \multicolumn{2}{|c|}{ Bundesliga } & \multirow{2}{*}{\multicolumn{2}{|c|}{$\begin{array}{ll} & \text { Liga } \\
0.380 & \pm 0.130\end{array}$}} & \multicolumn{2}{|c|}{ Serie A } \\
\hline & 0.334 & \pm 0.141 & & & 0.340 & \pm 0.123 \\
\hline SxGM & 0.381 & \pm 0.219 & 0.401 & \pm 0.204 & 0.325 & \pm 0.187 \\
\hline AxGM & 0.283 & \pm 0.134 & 0.311 & \pm 0.112 & 0.295 & \pm 0.113 \\
\hline 538 & 0.297 & \pm 0.166 & 0.303 & \pm 0.144 & 0.307 & \pm 0.135 \\
\hline LV & 0.342 & \pm 0.199 & 0.339 & \pm 0.215 & 0.311 & \pm 0.147 \\
\hline
\end{tabular}

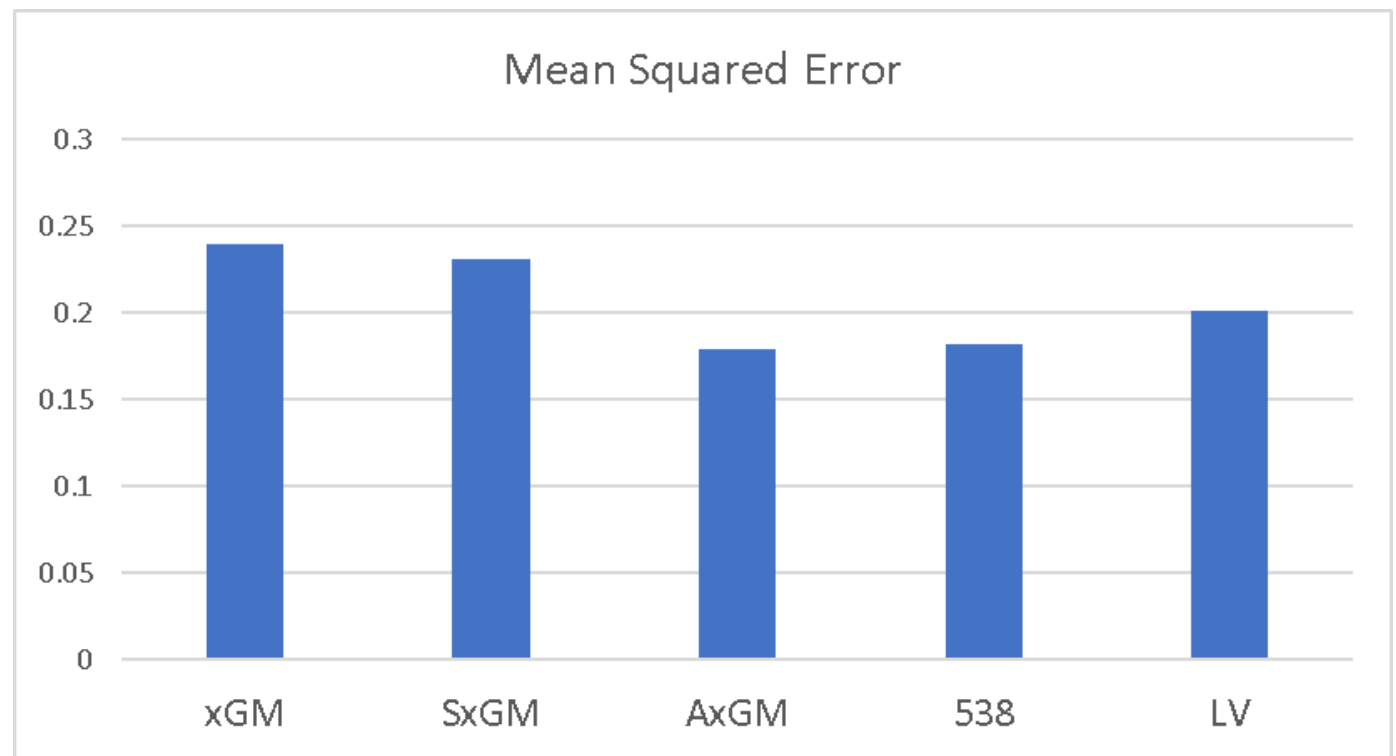

Figure 4 - Mean squared error results for our three models and the two gold standards. 
The third comparison we conducted between our predictive models and 538 were analyses of profitability. Three separate betting strategies were investigated for our models to measure and compare profitability between each other and against the gold standard 538. Net profits/losses together with accuracy across the 310 games in our dataset are shown in Table 2. The numbers in Table 2 assume that a $\$ 100$ bet was placed on each game that satisfied the betting strategy. Because each model and betting strategy had different probability thresholds for placing a bet on a game or not, the $\mathrm{N}$ is expressed as a range for the $>50$ and $>70$ betting strategies. The first number represents the lowest number of games any model following the $>50$ betting strategy bet on and the second number likewise represents the highest number of games bet on.

Table 2 - Accuracy and net profits/losses of each of our models separated across three betting strategies. The bolded values represent the best result for each column.

\begin{tabular}{ccccccc}
\multicolumn{2}{c}{ Best $(\mathrm{N}=310)$} & \multicolumn{2}{c}{$>50(\mathrm{~N}=104-213)$} & \multicolumn{2}{c}{$>70(\mathrm{~N}=3-70)$} \\
\hline \hline & Accuracy & Profit/Loss & Accuracy & Profit/Loss & Accuracy & Profit/Loss \\
xGM & $49.35 \%$ & $\mathbf{- \$ 2 , 6 2 8 . 1 4}$ & $57.69 \%$ & $-\$ 1,611.40$ & $66.67 \%$ & $\mathbf{\$ 7 5 2 . 6 7}$ \\
SxGM & $48.39 \%$ & $-\$ 4,789.11$ & $49.77 \%$ & $-\$ 5,279.70$ & $60.00 \%$ & $-\$ 746.71$ \\
AxGM & $50.00 \%$ & $-\$ 3,911.93$ & $\mathbf{6 0 . 7 4 \%}$ & $\mathbf{- \$ 1 , 3 5 2 . 2 0}$ & $\mathbf{7 0 . 0 0 \%}$ & $\$ 567.00$ \\
538 & $\mathbf{5 0 . 6 5 \%}$ & $-\$ 4,658.95$ & $60.54 \%$ & $-\$ 2,798.64$ & $65.71 \%$ & $-\$ 202.34$ \\
\hline
\end{tabular}

Betting on every match using the highest predicted probability resulted in nearly identical accuracies with SxGM being the least accurate but only with a $2.26 \%$ difference between it and the most accurate predictor 538. SxGM is the least accurate regardless of the betting strategy when compared to either xGM or AxGM. SxGM also reported the highest losses out of any of our models irrespective of betting strategy as well. xGM provided the highest profits out of every model for the best and $>70$ betting strategies despite it turning out a lower accuracy than AxGM. The reason for xGM's profitability is due to overpredicting away wins which usually are riskier and deal higher payouts.

Our models were profitable only when betting on match outcomes with a probability greater than $70 \%$. The superiority of the $>70$ betting strategy should come as no surprise given that only placing a bet on match outcomes that are highly likely to occur will reduce the amount of losses than placing a bet on every match regardless of how likely an outcome is. We see the accuracies of all our models are in line with and sometimes surpassing the accuracies found by 538. However, 538 is consistently losing money and this is probably due to its overuse of external factors such as team ranking in its prediction calculations. As a result, 538 is less likely to predict away and underdog wins. These results support our hypothesis that the introduction of too much complexity in a predictive algorithm can hinder its performance.

\section{Conclusions}

This study shows that using publicly available data combined with straightforward modeling steps lead to results that are comparable with the more complex gold-standards used in this study. We chose these gold-standards because of their availability as well as their popularity (538) and intuitive connection to betting (LV). It would be interesting to compare our models to more sophisticated ones involving Machine Learning algorithms such as Artificial Neural Networks. Our dataset did not span the whole seasons of the three Football Leagues due to the COVID-19 pandemic and some random data collection issues. It would be important to apply these models for a whole season to confirm these results and even identify temporal trends. The football season has various events (e.g., national team breaks, winter breaks) happening that can significantly alter team performance.

The predictive capabilities of our models were strong enough to be profitable under certain conditions. A further analysis of profitability in football betting could involve the investigation of the accuracy necessary to be 
profitable when betting all of the games as well as the use of parlays (e.g., betting multiple games at once) as betting strategy.

It would be interesting to improve the AxGM model by expanding its incorporation of home-field advantage and adding a team's defensive abilities in a more meaningful way than SxGM. Implementing these changes will require the rigorous use of more advanced modeling approaches such as regression and was beyond the goals of this study.

\section{References}

Cintia, P., Giannotti, F., Pappalardo, L., Pedreschi, D., \& Malvaldi, M. (2015, October). The harsh rule of the goals: Data-driven performance indicators for football teams. In 2015 IEEE International Conference on Data Science and Advanced Analytics (DSAA) (pp. 1-10). IEEE.

Cumming, G. (2014). The new statistics: Why and how. Psychological science, 25(1), 7-29.

Dobson, S., Goddard, J. A., \& Dobson, S. (2001). The economics of football (pp. 106-130). Cambridge: Cambridge University Press.

Eggels, H. P., van Elk, R., \& Pechenizkiy, M. (2016). Expected goals in soccer: Explaining match results using predictive analytics. In The machine learning and data mining for sports analytics workshop (p. 16).

Hucaljuk, J., \& Rakipovic, A. (2011). Predicting football scores using machine learning techniques. 2011 Proceedings of the 34th International Convention MIPRO, 1623-1627.

Karlis, D., \& Ntzoufras, I. (2003). Analysis of sports data by using bivariate Poisson models. Journal of the Royal Statistical Society: Series D (The Statistician), 52(3), 381-393.

Kramer, Adam. "Inside Look at How Las Vegas Oddsmakers Come Up with College Football Spread." Bleacher Report, Bleacher Report, 3 Oct. 2017, bleacherreport.com/articles/2104561-inside-look-at-how-las-vegas-oddsmakers-come-up-with-college-footballspread.

Jayboice. (2018, August 10). How Our Club Soccer Predictions Work. Retrieved from https://fivethirtyeight.com/methodology/how-our-club-soccer-predictions-work/

Lasek, J., Szlávik, Z., \& Bhulai, S. (2013). The predictive power of ranking systems in association football. International Journal of Applied Pattern Recognition, 1(1), 27-46.

Link, D. (2018). Data analytics in professional soccer. Springer Vieweg, Wiesbaden.

Macdonald, B. (2012, March). An expected goals model for evaluating NHL teams and players.

In Proceedings of the 2012 MIT Sloan Sports Analytics Conference,

http://www.sloansportsconference.com.

Mondello, M., \& Kamke, C. (2014). The introduction and application of sports analytics in professional sport organizations. Journal of Applied Sport Management, 6(2). 
Monks, J., \& Husch, J. (2009). The impact of seeding, home continent, and hosting on FIFA World Cup results. Journal of Sports Economics, 10(4), 391-408.

Pearson, A. (2017). How real-time analytics changes the face of the sports betting industry. Journal of Digital \& Social Media Marketing, 5(1), 61-75

Rein, R., \& Memmert, D. (2016). Big data and tactical analysis in elite soccer: future challenges and opportunities for sports science. SpringerPlus, 5(1), 1-13. 